\title{
THE STEADY PROFILE OF AN AXISYMMETRIG ICE SHEET
}

\author{
By I. R. Johnson \\ (School of Mathematics and Physics, University of East Anglia, Norwich NR4 7 TJ, England)
}

\begin{abstract}
ABSTRACr. Steady plane flow under gravity of an axisymmetric ice sheet resting on a horizontal rigid bed, subject to surface accumulation and ablation, basal drainage, and basal sliding is treated according to a power law between shear traction and velocity. The surface accumulation is taken to depend on height, and the drainage and sliding coefficient also depend on the height of overlying ice. The ice is described as a general non-linearly viscous incompressible fluid, and temperature variation through the ice sheet is neglected. Illustrations are presented for Glen's power law (including the special case of a Newtonian fluid), and the polynomial law of Colbeck and Evans. The analysis follows that of Morland and Johnson (1980) where the analogous problem for an ice sheet deforming under plane flow was considered. Comparisons are made between the two models and it is found that the effect of the third dimension is to reduce (or leave unchanged) the aspect ratio for the cases considered, although no general formula can be obtained. This reduction is
\end{abstract}

RÉsumé. Profil d'équilibre d'une calotte glaciaire ayant un axe de symétrie. On traite de l'écoulement plan permanent sous l'influence de la gravité d'une calotte glaciaire présentant un axe de symétrie reposant sur un lit rigide horizontal, soumis en surface à une accumulation et une ablation, une évacuation au fond et un glissement sur le lit suivant une loi-puissance pour la relation vitesse/cisaillement. L'accumulation en surface a été choisie comme liée à la hauteur, les coefficients d'écoulement et de glissement dépendent également de la hauteur de la glace susjacente. La glace est considérée comme un fluide incompressible non linéairement visqueux et on néglige la variation de la température à l'intérieur de la glace. On prescrit des illustrations correspondant à la loi-puissance de Glen (y compris le cas particulier du fluide Newtonien) et à la loi polynomiale de Colbeck et Evans. L'analyse fait suite à celle de Morland et Johnson (1980) où un problème analogue a été abordé pour une calotte glaciaire se déformant par un écoulement plan. On fait des comparaisons entre les deux modèles et on trouve que l'effet de la troisième dimension est de réduire (ou de laisser inchangé) le rapport de relief dans les cas considérés bien qu'on ne puisse pas obtenir de formule générale. On considère que cette réduction dépend à la fois de l'accumulation en surface et de la loi de glissement.

Zusammenfassung. Das stationäre Profil eines achssymetrischen Eisschildes. Es wird der stationäre, ebene Fluss unter Schwerkraft eines achssymetrischen Eisschildes, der auf einem horizontalen, starren Untergrund ruht und sowohl Akkumulation und Ablation an der Oberfläche, als auch Abfluss und Gleiten am Untergrund erfährt, nach einem Potenzgesetz für die Geschwindigkeit bei Scherung und Zug behandelt. Für die Akkumulation an der Oberfläche wird Abhängigkeit von der Höhe angenommen; die Koeffizienten für Abfluss und Gleiten hängen von der Höhe des überlagernden Eises ab. Das Eis wird als eine allgemeine, nicht-linear viskose, unkomprimierbare Flüssigkeit beschrieben; Temperaturschwankungen im Eisschild werden vernachlässigt. Darstellungen beziehen sich auf Glen's Potenzgesetzt (einschliesslich des Sonderfalls einer Newton'schen Flüssigkeit) und auf das Polynomgesetz von Colbeck und Evans. Die Analyse folgt der von Morland und Johnson (1980), wo das analogue Problem für einen Eisschild, der sich unter ebenem Fluss verformt, betrachtet wurde. Zwischen den beiden Modellen werden Vergleiche gezogen, wobei sich feststellen lässt, dass die Einbeziehung der dritten Dimension eine Reduktion (bzw. keine Veränderung) des Umrissverhältnisses der beiden betrachteten Fälle zur Folge hat, obwohl keine allgemeine Formel zu gewinnen ist. Diese Reduktion erweist sich als abhängig sowohl von der Oberflächenakkumulation wie vom Gleitgesetz.

\section{InTRODUGTION}

The mechanics of a bounded ice sheet with steady free surface has been considered by Nye (1959), Weertman (1961), and more recently by Morland and Johnson (1980). Nye considered both plane and axisymmetric flow in order to estimate the effects of a third dimension in comparison with the plane-flow restriction. Morland and Johnson's analysis was for an ice sheet deforming in plane flow only, solving the full momentum equations by a regular perturbation technique. The equivalent axisymmetric solution is now presented. There are large similarities between the plane and axisymmetric analyses and details which apply to both problems are not repeated. Henceforth Morland and Johnson (1980) will be referred to as $\mathrm{M}$ and $\mathrm{J}$.

Consider an ice sheet shown by the cross-section in Figure I, with horizontal bed $z=0$ and surface $z=h(r)$ in cylindrical polar coordinates $(r, \theta, z)$, and all physical variables independent of $\theta$. The velocity components are $(u, \mathrm{o}, w)$ and $g$ is the constant acceleration due to gravity. $\tau$ is the Cauchy stress tensor with $\sigma_{r \theta}=\sigma_{r z}=0$, and $\left(t_{\mathrm{n}}, t_{\mathrm{s}}\right)$ denote normal and tangential tractions on the surface. The ice sheet is maintained in steady flow by surface accumulation and ablation and basal drainage, with zero net flux. 


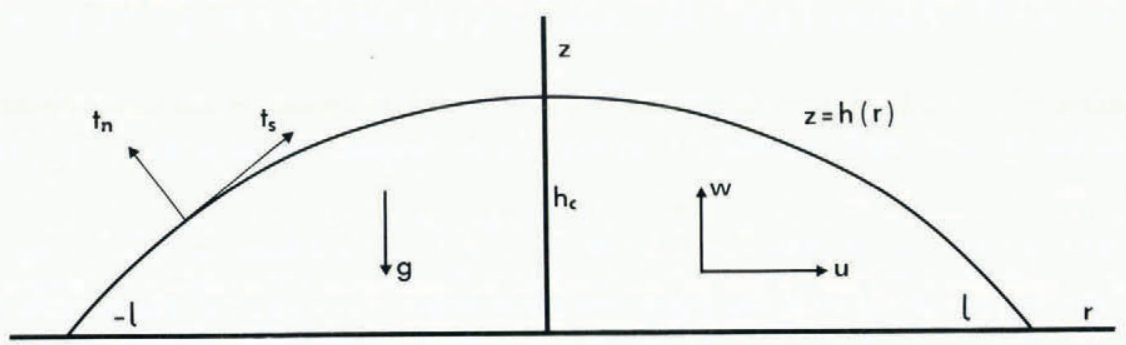

Fig. I. Ice-sheet cross-section.

By considering constant surface accumulation Nye (1959) obtained the solution

$$
\left(\frac{h}{h_{\mathrm{c}}}\right)^{2+\mathrm{I} / m}+\left(\frac{r}{l}\right)^{\mathrm{I}+\mathrm{I} / m}=(\mathrm{I})
$$

for the profile, where

$$
\rho g(m+\mathrm{I}) h_{\mathrm{c}^{2+\mathrm{I} / m}}=A\left(\frac{q}{2}\right)^{\mathrm{I} / m}(2 m+\mathrm{I}) l^{\mathrm{I}+\mathrm{I} / m},
$$

and $A$ and $m$ are constants appearing in the basal sliding law

$$
u=u(r)=\left\{\sigma_{r z}(z=0) / A\right\}^{m} .
$$

$q$ is the constant accumulation, $\rho$ is the uniform density of ice, $h_{\mathrm{c}}$ is the height at the centre of the ice sheet, and $l$ the radius.

As for the plane-flow profile, the condition of small surface slope is violated in the axisymmetric profile except in some central zone. Nye's analysis is independent of the flow law and any temperature dependence. He has, nevertheless, deduced that observed temperature profiles give rise to an approximately uniform horizontal velocity profile above a thin bottom layer, and used this as the basic kinematic approximation. The analysis in this paper does not include any temperature variation so that Nye's deduction cannot be tested, nor can any effect of temperature variation be predicted.

Following the approach of $\mathrm{M}$ and $\mathrm{J}$, for the axisymmetric problem the boundary conditions are

$$
\begin{aligned}
z & =0: & \sigma_{r z} & =\lambda(h)(u)^{\mathrm{I} / m}, \\
z & =0: & w & =-b(h) \leqslant 0,
\end{aligned}
$$

where the basal drainage $b$ and the sliding coefficient $\lambda$ depend on the overlying height of ice. If $q$ is the accumulation (volume flux per unit horizontal area) referred to the horizontal cross-section, then

$$
z=h(r): \quad w-h^{\prime} u=-q(h) .
$$

The free-surface conditions are

$$
\begin{aligned}
\left(\mathrm{I}+\gamma^{2}\right)\left(\sigma_{r r}+\sigma_{z z}\right) & +\left(\mathrm{I}-\gamma^{2}\right)\left(\sigma_{z z}-\sigma_{r r}\right)+4 \gamma \sigma_{r z}=0, \\
-\left(\mathrm{I}-\gamma^{2}\right) \sigma_{r z}+\gamma\left(\sigma_{z z}-\sigma_{r r}\right) & =0,
\end{aligned}
$$

and symmetry requires

$$
r=0: \quad u=0, \quad \sigma_{r z}=0, \quad h^{\prime}=\gamma=0 .
$$

Formulation of the full problem in dimensionless variables introduces a small parameter $\nu$. However, the approximation $\nu=0$ does not permit a bounded ice sheet. Rescaling the horizontal coordinate by a small factor $\epsilon(\nu) \ll I$ allows a regular perturbation solution in $\epsilon$. $\nu$ and $\epsilon$ are identical to the parameters which arose in the plane-flow analysis, and again $\epsilon$ 
defines the small magnitude of the surface slope. The small-slope solution is valid up to the margin $(h=0)$ provided the sliding coefficient $\lambda(h) \simeq \lambda_{0} h$ as $h \rightarrow 0$, as for the plane flow case.

The results obtained are compared with those for the plane-flow solution ( $\mathrm{M}$ and $\mathrm{J}$ ) for the case $m=\mathbf{I}$. In all illustrations it is found that the aspect ratio decreases (or is unchanged), as predicted by Nye (1959), but here the results demonstrate the dependence of the decrease on the accumulation rate and the sliding law.

2. Balance laws AND constitutive equations

The ice is assumed to be incompressible, so mass balance requires

$$
r \frac{\partial w}{\partial z}+\frac{\partial}{\partial r}(r u)=0
$$

Inertia terms are negligible in this slow viscous flow so momentum balance requires

$$
\left.\begin{array}{c}
\frac{\partial \sigma_{r r}}{\partial r}+\frac{\partial \sigma_{r z}}{\partial z}+\frac{\sigma_{r r}-\sigma_{\theta \theta}}{r}=0 \\
\frac{\partial \sigma_{r z}}{\partial r}+\frac{\partial \sigma_{z z}}{\partial z}+\frac{\sigma_{r z}}{r}-\rho g=0 .
\end{array}\right\}
$$

Following $\mathrm{M}$ and $\mathrm{J}$, the ice is assumed to be an incompressible non-linear viscous fluid with a temperature-dependent rate factor (Morland, I979), so

$$
\frac{\boldsymbol{\sigma}+p \mathbf{r}}{\sigma_{0}}=\phi_{1}\left(\hat{I}_{2}, \hat{I}_{3}\right) \hat{\mathbf{D}}+\phi_{2}\left(\hat{I}_{2}, \hat{I}_{3}\right)\left[\hat{\mathbf{D}}^{2}-\frac{2}{3} \hat{I}_{2} \mathbf{r}\right],
$$

where

$$
p=-\frac{1}{3} \operatorname{tr} \sigma, \quad i_{2}=\frac{1}{2} \operatorname{tr} \hat{\mathbf{D}}^{2}, \quad i_{3}=\operatorname{det} \hat{\mathbf{D}},
$$

and $\hat{\mathbf{D}}=\mathbf{D} / D_{0} a(T)$. The non-zero components of $\mathbf{D}$ are

$$
\left.\begin{array}{c}
D_{r r}=\frac{\partial u}{\partial r}, \quad D_{\theta \theta}=\frac{u}{r}, \quad D_{z z}=\frac{\partial w}{\partial z}, \\
D_{r z}=D_{z r}=\frac{\mathrm{I}}{2}\left(\frac{\partial u}{\partial z}+\frac{\partial w}{\partial r}\right),
\end{array}\right\}
$$

$T$ denotes temperature, and $a(T)$ is the rate factor, normalized by $a\left(T_{0}\right)=\mathrm{I}$ for some temperature $T_{0}$, with $a^{\prime}(T) \geqslant 0$. $\sigma_{0}$ and $D_{0}$ denote a constant stress magnitude and a constant strain-rate magnitude respectively, so that $\hat{\mathbf{D}}$, the invariants $\hat{I}_{2}, \hat{I}_{3}$, and the response functions $\phi_{1}, \phi_{2}$ are dimensionless.

The more commonly adopted laws for $\mathbf{D}$ give the simpler form

$$
\hat{\mathbf{D}}=\omega\left(\mathcal{F}_{2}\right) \frac{\boldsymbol{\sigma}+p \mathbf{r}}{\sigma_{0}}, \quad \mathcal{F}_{2}=\frac{\mathrm{I}}{2} \operatorname{tr}\left(\frac{\boldsymbol{\sigma}+p \mathbf{r}}{\sigma_{0}}\right)^{2},
$$

which implies

With

$$
\left.\begin{array}{c}
\tilde{I}_{2}=\mathcal{J}_{2} \omega^{2}\left(\mathcal{J}_{2}\right)=G\left(\mathcal{J}_{2}\right) \text { say, } \quad \phi_{2} \equiv 0, \\
\phi_{1}=\phi_{\mathrm{I}}\left(I_{2}\right), \quad \mathcal{J}_{2}=I_{2} \phi_{1}{ }^{2}\left(I_{2}\right)=G^{-1}\left(I_{2}\right) .
\end{array}\right\}
$$

$$
\sigma_{0}=10^{5} \mathrm{~N} \mathrm{~m}^{-2}, \quad D_{0}^{-1}=3 \times 10^{7} \mathrm{~s}, \quad T_{0}=273 \mathrm{~K},
$$


Glen's law gives

$$
\left.\begin{array}{c}
\omega\left(\mathcal{F}_{2}\right)=\mathrm{I} \cdot 5 k\left(3 \mathcal{F}_{2}\right)^{(n-1) / 2}, \quad \phi_{1}\left(\hat{I}_{2}\right)=\frac{2}{3} k^{-1 / n}\left(\frac{4}{3} \hat{I}_{2}\right)^{-(n-1) / 2 n}, \\
k=0.17, \quad n=\mathrm{I} \cdot 3 \rightarrow 4,
\end{array}\right\}
$$

and the Colbeck and Evans polynomial law gives

$$
\left.\begin{array}{c}
\omega\left(\mathcal{F}_{2}\right)=\mathrm{I} .5\left(C_{0}+{ }_{3} C_{1} \mathcal{F}_{2}+9 C_{2} \mathcal{J}_{2}{ }^{2}\right), \\
C_{0}=0.2 \mathrm{I}, \quad C_{1}=0.14, \quad C_{2}=0.055 .
\end{array}\right\}
$$

In the following analysis it is assumed that in the general law (10) the stress contribution from a non-zero $\phi_{2}$ term is not of greater magnitude than that of the $\phi_{1}$ term. It is also assumed that $\left|\phi_{2}\right| \leqslant O(\mathrm{I})$, but the singularity in $\phi_{1}$ arising from Glen's law (Equation (I6)) will be considered.

\section{Dimensionless formulation AND the SMAll parameter}

Introduce dimensionless variables by

$$
\left.\begin{array}{c}
(r, z, h, l)=h_{0}(R, Z, H, L), \quad(\boldsymbol{\sigma}, p)=\rho g h_{0}(\boldsymbol{\Sigma}, P), \\
(u, w, q, b)=q_{\mathrm{m}}(U, W, Q, B),
\end{array}\right\}
$$

where $h_{0}$ is a magnitude of the maximum ice thickness and $q_{\mathrm{m}}$ is a magnitude of maximum accumulation density. Take $q_{\mathrm{m}}=q\left(h_{0}\right)$ on the assumption that ablation at lower heights does not significantly exceed this value. It is supposed that the drainage magnitude is not greater than that of $Q$. Define

Setting

$$
h^{\prime}(r)=H^{\prime}(R)=\Gamma(H) .
$$

$$
U=\frac{\mathrm{I}}{R} \frac{\partial \Psi}{\partial Z}, \quad W=-\frac{\mathrm{I}}{R} \frac{\partial \Psi}{\partial R},
$$

in terms of a dimensionless stream function $\Psi(r, z)$, satisfies the mass-balance equation (8). The momentum equations (9) become

$$
\left.\begin{array}{c}
\frac{\partial \Sigma_{r r}}{\partial R}+\frac{\partial \Sigma_{r z}}{\partial Z}+\frac{\Sigma_{r r}-\Sigma_{\theta \theta}}{R}=0, \\
\frac{\partial \Sigma_{r z}}{\partial R}+\frac{\partial \Sigma_{z z}}{\partial Z}+\frac{\Sigma_{r z}}{R}-\mathrm{I}=0 .
\end{array}\right\}
$$

Boundary conditions $(2)-(7)$ become, for $z=0$ :

$$
\begin{gathered}
\Sigma_{r z}=\Lambda(H)\left[\frac{\mathrm{I}}{R} \frac{\partial \Psi}{\partial Z}\right]^{\mathrm{I} / m}, \quad \Lambda(H)=\frac{\lambda(h) q_{\mathrm{m}}{ }^{\mathrm{I} / m}}{\rho g h_{0}}, \\
\frac{\mathrm{I}}{R} \frac{\partial \Psi}{\partial R}=B(H) ;
\end{gathered}
$$

for $z=H(R)$ :

$$
\begin{aligned}
\frac{\mathrm{I}}{R} \frac{\partial \Psi}{\partial R}+\frac{\Gamma}{R} \frac{\partial \Psi}{\partial Z} & =Q(H), \\
\left(\mathrm{I}+\Gamma^{2}\right)\left(\Sigma_{r r}+\Sigma_{z z}\right)+\left(\mathrm{I}-\Gamma^{2}\right)\left(\Sigma_{z z}-\Sigma_{r r}\right)+4 \Gamma \Sigma_{r z} & =0, \\
-\left(\mathrm{I}-\Gamma^{2}\right) \Sigma_{r z}+\Gamma\left(\Sigma_{z z}-\Sigma_{r r}\right) & =0 ;
\end{aligned}
$$


and for $R=0$ :

$$
U=0 \Rightarrow \lim _{R \rightarrow 0} \frac{\mathrm{I}}{R} \frac{\partial \Psi}{\partial Z}=0, \quad \Sigma_{r z}=0, \quad \Gamma=0 .
$$

The tensors $\hat{\mathbf{D}}, \hat{\mathbf{D}}^{2}$ have non-zero components

$$
\begin{gathered}
\hat{D}_{r r}=\delta \frac{\partial}{\partial R}\left(\frac{\mathrm{I}}{R} \frac{\partial \Psi}{\partial Z}\right), \quad \hat{D}_{\theta \theta}=\delta \frac{\mathrm{I}}{R^{2}} \frac{\partial \Psi}{\partial Z}, \quad \hat{D}_{z z}=-\delta \frac{\partial}{\partial Z}\left(\frac{\mathrm{I}}{R} \frac{\partial \Psi}{\partial R}\right), \\
\hat{D}_{r z}=\frac{\delta}{2}\left\{\frac{\partial}{\partial Z}\left(\frac{\mathrm{I}}{R} \frac{\partial \Psi}{\partial Z}\right)-\frac{\partial}{\partial R}\left(\frac{\mathrm{I}}{R} \frac{\partial \Psi}{\partial R}\right)\right\}=\frac{\delta}{2}\left\{\frac{\mathrm{I}}{R}\left(\frac{\partial^{2} \Psi}{\partial Z^{2}}-\frac{\partial^{2} \Psi}{\partial R^{2}}\right)+\frac{\mathrm{I}}{R^{2}} \frac{\partial \Psi}{\partial R}\right\}, \\
\left(\hat{\mathbf{D}}^{2}\right)_{r r}=\delta^{2}\left[\left\{\frac{\partial}{\partial R}\left(\frac{\mathrm{I}}{R} \frac{\partial \Psi}{\partial Z}\right)\right\}^{2}+\frac{\mathrm{I}}{4}\left\{\frac{\partial}{\partial Z}\left(\frac{\mathrm{I}}{R} \frac{\partial \Psi}{\partial Z}\right)-\frac{\partial}{\partial R}\left(\frac{\mathrm{I}}{R} \frac{\partial \Psi}{\partial R}\right)\right\}^{2}\right], \\
\left(\hat{\mathbf{D}}^{2}\right)_{\theta \theta}=\delta^{2} \frac{\mathrm{I}}{R^{4}}\left(\frac{\partial \Psi}{\partial Z}\right)^{2}, \\
\left(\hat{\mathbf{D}}^{2}\right)_{z z}=\delta^{2}\left[\left\{\frac{\partial}{\partial Z}\left(\frac{\mathrm{I}}{R} \frac{\partial \Psi}{\partial R}\right)\right\}^{2}+\frac{\mathrm{I}}{4}\left\{\frac{\partial}{\partial Z}\left(\frac{\mathrm{I}}{R} \frac{\partial \Psi}{\partial Z}\right)-\frac{\partial}{\partial R}\left(\frac{\mathrm{I}}{R} \frac{\partial \Psi}{\partial R}\right)\right\}^{2}\right], \\
\left(\hat{\mathbf{D}}^{2}\right)_{r z}=\frac{\delta^{2}}{2}\left\{\frac{\partial}{\partial Z}\left(\frac{\mathrm{I}}{R} \frac{\partial \Psi}{\partial Z}\right)-\frac{\partial}{\partial R}\left(\frac{\mathrm{I}}{R} \frac{\partial \Psi}{\partial R}\right)\right\}\left\{\frac{\partial}{\partial R}\left(\frac{\mathrm{I}}{R} \frac{\partial \Psi}{\partial Z}\right)-\frac{\partial}{\partial Z}\left(\frac{\mathrm{I}}{R} \frac{\partial \Psi}{\partial R}\right)\right\}, \\
I_{2}=\delta^{2}\left[\left\{\frac{\partial}{\partial R}\left(\frac{\mathrm{I}}{R} \frac{\partial \Psi}{\partial Z}\right)\right\}^{2}+\left(\frac{\mathrm{I}}{R^{2}} \frac{\partial \Psi}{\partial Z}\right)^{2}+\frac{\mathrm{I}}{4}\left\{\frac{\partial}{\partial Z}\left(\frac{\mathrm{I}}{R} \frac{\partial \Psi}{\partial Z}\right)-\frac{\partial}{\partial R}\left(\frac{\mathrm{I}}{R} \frac{\partial \Psi}{\partial R}\right)\right\}^{2}+\right. \\
\delta=q_{\mathrm{m}} / a D_{0} h_{0},
\end{gathered}
$$

where, under typical conditions ( $M$ and $J)$,

$$
\mathrm{IO}^{-2}>\delta>3 \times \mathrm{IO}^{-4} \text {. }
$$

To deal with the singularity in $\phi_{\mathrm{I}}$ at $\hat{I}_{2}=0$ for Glen's law (see $\mathrm{M}$ and $\mathrm{J}$ ), let

so that

$$
\phi_{1} \hat{\mathbf{D}}=\phi_{1}\left(\hat{\mathbf{D}} \hat{I}_{2}^{-\alpha}\right), \quad 0 \leqslant \alpha<\frac{1}{2},
$$

is bounded, and for Glen's law

$$
\phi_{1}=i_{2}^{\alpha} \phi_{1}
$$

$$
\alpha=\frac{n-\mathrm{I}}{2 n}<\frac{\mathrm{I}}{2}, \quad \phi_{\mathrm{I}}=\frac{2}{3}\left(\frac{3}{4}\right)^{(n-\mathrm{I}) / 2 n} k^{-\mathrm{I} / n}=O(\mathrm{I}),
$$

and $\phi_{\mathrm{I}}$ is constant, while the finite viscosity case is obtained by setting $\alpha=0$, with $\phi_{\mathrm{I}}=O(\mathrm{r})$. Hence Equations (3O) and (3I) cover both the cases with a power-law singularity and those which are non-singular. Equation (10) is now

where $\hat{\mathbf{D}}^{2}=\delta^{2} \overline{\mathbf{D}}$ and

$$
\boldsymbol{\Sigma}+P \mathbf{I}=\nu\left[\phi_{1} I_{2}^{-\alpha} \overline{\mathbf{D}}+\delta^{1+2 \alpha} \phi_{2}\left\{\overline{\mathbf{D}}^{2}-\frac{2}{3} I_{2} \mathbf{I}\right\}\right],
$$

$$
\nu=s \delta^{\mathrm{I}-2 \alpha}, \quad s=\sigma_{0} / \rho g h_{0} .
$$

The parameters $\delta, \nu$, and $s$ are identical to those of the plane-flow analysis and typically

$$
\nu \ll \mathrm{I} \text {, }
$$


(see table I, M and J), which is the condition adopted. Note also that

$$
\theta=\delta / s \leqslant O(\mathbf{I}),
$$

for practical conditions.

By setting $\nu=0$ in the momentum equations a uniform parallel slab of infinite extent is predicted with

$$
P=H-Z, \quad H=\text { constant, }
$$

excluding a margin at finite radius.

\section{Scaled variables and slope magnitude}

For a non-zero surface slope, $P$, to leading order, must not be independent of $R$, so the horizontal momentum balance given by the first of Equations (2I) must involve the shear stress gradient in $Z$. This suggests a horizontal coordinate contraction

$$
\rho=\epsilon R, \quad \Gamma=\epsilon \gamma=\epsilon \frac{\mathrm{d} H}{\mathrm{~d} \rho},
$$

(cf. $\mathrm{M}$ and $\mathrm{J}$ ), where $\rho, \gamma=O(\mathrm{I})$ and $\Gamma$ has magnitude $\epsilon$. To retain the surface accumulation balance given by Equation (24), a stream-function scaling

$$
\psi=\epsilon^{2} \Psi=O(\mathrm{I})
$$

is required, so Equation (24) becomes

$$
Z=H: \quad \frac{\mathrm{I}}{\rho} \frac{\partial \psi}{\partial \rho}+\frac{\gamma}{\rho} \frac{\partial \psi}{\partial Z}=Q .
$$

Note that for plane flow $\Psi$ was scaled by $\epsilon$ but the relations between the velocity components and the axisymmetric stream function are different.

As before, a balance in the momentum equations requires $\epsilon \ll \mathrm{I}$, and only leading-order terms are presented for brevity. Thus, by Equations (28) and (33)

$$
\begin{gathered}
I_{2}=\epsilon^{-2} i ; \quad i \approx\left[\frac{\mathrm{I}}{2} \frac{\partial}{\partial Z}\left(\frac{\mathrm{I}}{\rho} \frac{\partial \psi}{\partial Z}\right)\right]^{2}=O(\mathrm{I}), \\
\left(\begin{array}{c}
\Sigma_{r r} \\
\Sigma_{z z}
\end{array}\right) \simeq-P+\nu\left[ \pm \phi_{1} \epsilon^{2 \alpha} i^{-\alpha} \frac{\partial}{\partial \rho}\left(\frac{\mathrm{I}}{\rho} \frac{\partial \psi}{\partial Z}\right)+\frac{\mathrm{I}}{3} \epsilon^{-2} \delta^{\mathrm{I}+2 \alpha} \phi_{2} i\right], \\
\Sigma_{\theta \theta} \simeq-P+\nu\left[\phi_{1} \epsilon^{2 \alpha} i^{-\alpha} \frac{\mathrm{I}}{\rho^{2}} \frac{\partial \psi}{\partial Z}-\frac{2}{3} \epsilon^{-2} \delta^{\mathrm{I}+2 \alpha} \phi_{2} i\right], \\
\Sigma_{r z} \simeq \nu\left[\frac{\mathrm{I}}{2} \phi_{1} \epsilon^{2 \alpha-\mathrm{I}} i^{-\alpha} \frac{\partial}{\partial Z}\left(\frac{\mathrm{I}}{\rho} \frac{\partial \psi}{\partial Z}\right)-\frac{2}{3} \epsilon^{-2} \delta^{\mathrm{I}+2 \alpha} \phi_{2} i\right] .
\end{gathered}
$$

The balance now requires

$$
\nu \epsilon^{2 \alpha-2}=O(\mathrm{I}), \quad \nu \epsilon^{-2} \delta^{2 \alpha+1} \leqslant O(\mathrm{I}),
$$

and hence

$$
\epsilon=\nu^{1 /(2-2 \alpha)}=\nu^{n /(n+1)}=\delta \theta^{-n /(n+1)} .
$$

This restriction is identical to that in the plane-flow case so the $\phi_{2}$ term does not contribute to $\boldsymbol{\Sigma}$ to leading order with the assumption $\left|\phi_{2}\right| \leqslant O(\mathrm{I})$. For most, if not all, practical conditions

$$
\epsilon \ll \mathbf{I},
$$

which is assumed here. 


\section{LeAding-ORDER APPROXIMATION} and

A power-series expansion in $\epsilon$ is again appropriate, so let $P=P_{0}+O(\epsilon), \psi=\psi_{0}+O(\epsilon)$,

$$
H=\eta(\rho)+O(\epsilon), \quad \gamma_{0}=\eta^{\prime}(\rho) .
$$

Denote all leading-order quantities by a subscript or superscript 0 , so

$$
\left.\begin{array}{c}
\Sigma_{r r^{0}}=\Sigma_{\theta \theta^{0}}=\Sigma_{z z^{0}}=-P_{0}, \\
\Sigma_{r z^{0}}=\frac{\mathrm{I}}{2} \epsilon \bar{\phi}_{1} i^{-\alpha} \frac{\partial}{\partial Z}\left(\frac{\mathrm{I}}{\rho} \frac{\partial \psi_{0}}{\partial Z}\right), \\
i_{0}=\left[\frac{\mathrm{I}}{2} \frac{\partial}{\partial Z}\left(\frac{\mathrm{I}}{\rho} \frac{\partial \psi_{0}}{\partial Z}\right)\right]^{2}, \\
U_{0}=\frac{\epsilon^{-1}}{\rho} \frac{\partial \psi_{0}}{\partial Z}, \quad W_{0}=-\frac{\mathrm{I}}{\rho} \frac{\partial \psi_{0}}{\partial \rho} .
\end{array}\right\}
$$

Proceeding with the solution as before gives rise to the ordinary differential equation

$$
\begin{aligned}
\frac{\mathrm{d}}{\mathrm{d} \rho}\left[\eta \rho\left(-\frac{\eta^{\prime} \eta}{j \bar{\Lambda}(\eta)}\right]^{m}-\frac{\eta \rho}{\eta^{\prime}} g_{\mathrm{I}}\left(-\eta^{\prime} \eta\right)-\frac{\rho}{\left(\eta^{\prime}\right)^{2}} g_{2}\left(-\eta^{\prime} \eta\right)\right) & =\rho\{Q(\eta)-B(\eta)\} \\
& =\rho Q^{\star}(\eta),
\end{aligned}
$$

for the free surface $H=\eta(\rho)$, where

$$
j=\theta^{(n-1)(m+\mathrm{I}) / 2 m(n+\mathrm{I})} \leqslant O(\mathrm{I}), \quad \bar{\Lambda}=\frac{\lambda q_{\mathrm{m}^{\mathrm{I}} / m}}{\rho g h_{0}}\left(\frac{\theta}{\delta^{2}}\right)^{(m+\mathrm{I}) / 2 m} .
$$

$\bar{\Lambda}$ is normalized on the scale $\epsilon_{\mathrm{I}}=\epsilon(n=\mathrm{I})$ and so is independent of $n$. The $g$ functions arising from the constitutive laws are unaltered, and so for Glen's law and the Colbeck and Evans polynomial laws respectively

and

$$
\left.\begin{array}{l}
g(t)=3^{\frac{1}{2}(n+1)} k t^{n}, \\
g(t)=3^{t}\left(C_{0}+3 C_{1} \theta t^{2}+9 C_{2} \theta^{2} t^{4}\right),
\end{array}\right\}
$$

$$
g_{\mathrm{I}}(t)=\int_{0}^{t} g\left(t^{\prime}\right) \mathrm{d} t^{\prime}, \quad g_{2}(t)=\int_{0}^{t} g_{\mathrm{I}}\left(t^{\prime}\right) \mathrm{d} t^{\prime} .
$$

Here the argument $-\eta^{\prime} \eta$ is always positive. $\psi_{0}$ is given by

$$
\psi_{0}=\frac{\rho}{\left(\eta^{\prime}\right)^{2}} g_{2}\left[-\eta^{\prime}(\eta-Z)\right]+Z f_{\mathrm{I}}(\rho)+f_{0}(\rho),
$$

where

$$
\left.\begin{array}{rl}
f_{0}^{\prime}(\rho) & =\rho B(\eta)-\frac{\partial}{\partial \rho}\left[\frac{\rho g_{2}\left(-\eta^{\prime} \eta\right)}{\left(\eta^{\prime}\right)^{2}}\right], \\
f_{\mathrm{I}}(\rho) & =\rho\left[-\frac{\eta^{\prime} \eta}{j \bar{\Lambda}}\right]^{m}-\frac{\rho}{\eta^{\prime}} g_{\mathrm{I}}\left(-\eta^{\prime} \eta\right) .
\end{array}\right\}
$$


The leading order stress components are

$$
\left.\begin{array}{c}
\Sigma_{r r^{0}}=\Sigma_{\theta \theta^{0}}=\Sigma_{z z^{0}}=-P_{0}=-\{\eta(\rho)-z\}, \\
\Sigma_{r z^{0}}=-\epsilon \eta^{\prime}(\rho)\{\eta(\rho)-z\},
\end{array}\right\}
$$

and these have the same form as for plane flow.

\section{General properties and validity}

Equation (48) is analogous to equation (68) in $\mathrm{M}$ and $\mathrm{J}$. It differs in the appearance of the $\rho$ term on both sides of the equation which leads to a stricter restriction on $\bar{\Lambda}(\eta)$ and also requires a slightly different numerical approach. Rewrite Equation (48) as a first-order ordinary differential equation for $\eta^{\prime}=\gamma_{0}(\eta)$ :

$$
\left.\begin{array}{c}
\gamma_{0} \frac{\mathrm{d}}{\mathrm{d} \eta}\left[\eta \rho\left(-\frac{\gamma_{0} \eta}{j \bar{\Lambda}}\right)^{m}+\eta^{2} \rho \Omega\left(-\gamma_{0} \eta\right)\right]=\rho Q^{\star}, \\
\Omega(X) \simeq \Omega_{0} X^{n} \text { as } X \rightarrow 0 .
\end{array}\right\}
$$

For Glen's law and Colbeck and Evans' law respectively

$$
\left.\begin{array}{l}
\Omega=\frac{3^{\frac{1}{2}(n+\mathrm{I})} k\left(-\gamma_{0} \eta\right)^{n}}{n+2}, \\
\Omega=C_{0}\left(-\gamma_{0} \eta\right)+\frac{9}{5} \theta C_{1}\left(-\gamma_{0} \eta\right)^{3}+\frac{27}{7} \theta^{2} C_{2}\left(-\gamma_{0} \eta\right)^{5} .
\end{array}\right\}
$$

Zero mass flux requires that, integrating over the surface and the bed,

$$
\int Q^{\star} \mathrm{d} S=\mathrm{o} \Rightarrow \int_{0}^{\epsilon R} Q^{\star} \rho \mathrm{d} \rho=\mathrm{o},
$$

which implies for $\eta \rightarrow 0$ :

$$
\left.\begin{array}{ll}
\gamma_{0}(\eta) \simeq-\gamma_{\mathrm{m}} \eta^{\beta}\left(\mathrm{I}-\gamma_{\mathrm{I}} \eta+\ldots\right), & \beta \geqslant 0, \\
\bar{\Lambda}(\eta) \simeq \lambda_{0} \eta^{t}\left(\mathrm{I}-\lambda_{\mathrm{I}} \eta+\ldots\right), & 0 \leqslant m t<\mathrm{I}+m(\mathrm{I}+\beta) .
\end{array}\right\}
$$

As $\eta \rightarrow \eta_{\mathrm{c}}=\eta(\mathrm{o}), \bar{\Lambda}$ and $Q^{\star}$ are finite and $\gamma_{0}, \rho \rightarrow 0$, so the essential behaviour of Equation (55) is

$$
\gamma_{0} \frac{\mathrm{d}}{\mathrm{d} \eta}\left(\rho \gamma_{0}^{l}\right) \simeq \rho
$$

where $l=\min (m, n)$. Hence

$$
\gamma_{0} \simeq\left(\eta_{\mathrm{c}}-\eta\right)^{\mathrm{I} /(l+1)}, \quad \rho \simeq\left(\eta_{\mathrm{c}}-\eta\right)^{l /(l+1)} .
$$

As $\eta \rightarrow 0(0 \leqslant z \leqslant \eta)$ or $\gamma_{0} \rightarrow 0$ the dominant terms of $\psi_{0}$, in both limits, from Equation (52), are

$$
\psi_{0}-\int_{0}^{\rho} \rho B(\eta) \mathrm{d} \rho \simeq \rho\left(-\gamma_{0}\right)^{n} \eta^{n+2} \quad \text { or } \quad \rho Z\left(-\frac{\gamma_{0} \eta}{\bar{\Lambda}}\right)^{m} .
$$

Thus, as $\gamma_{0} \rightarrow 0, \eta \rightarrow \eta_{c}$,

$$
\psi_{0} \simeq\left(\eta_{\mathrm{c}}-\eta\right)^{2 l /(l+1)}
$$


Direct differentiation of both terms in (6I) show that bounded $\frac{\partial}{\partial \rho}\left(\frac{\mathrm{I}}{\rho} \frac{\partial \psi_{0}}{\partial \rho}\right), \frac{\partial^{2}}{\partial \rho^{2}}\left(\frac{\mathrm{I}}{\rho} \frac{\partial \psi_{0}}{\partial \rho}\right)$ requires (i) if $l=n$ then $l=n=\mathrm{I}$ and $m=\mathrm{I}$ or 2 or $m \geqslant 3$; (ii) if $l=m$ then $l=m=\mathrm{I}$ and $n=\mathrm{I}$ or 2 or $n \geqslant 3$. These are the same restrictions as for plane flow.

It now remains to look at the behaviour of $\gamma_{0}$ as $\eta \rightarrow 0$ to find any restrictions on $t$ and $m$ to ensure that $\beta \geqslant 0$. Let

$$
Q^{\star}(\eta)=-Q_{0}\left(\mathrm{I}-q_{\mathrm{r}} \eta+\ldots\right) \quad \text { as } \eta \rightarrow \mathrm{o}, Q_{0}>0 .
$$

Since $\rho=\int \frac{\mathrm{d} \eta}{\gamma_{0}}$ then as $\eta \rightarrow 0$,

$$
\rho \simeq \rho_{\mathrm{m}}-\frac{\mathrm{I}}{\gamma_{\mathrm{m}}}\left[\frac{\eta^{\mathrm{I}-\beta}}{\mathrm{I}-\beta}+\frac{\gamma_{\mathrm{I}} \eta^{2-\beta}}{2-\beta}\right] .
$$

Substituting these asymptotic values in Equation (55) and balancing both sides of the equation leads to

$$
\beta=\frac{m(t-\mathrm{I})}{\mathrm{I}+m}=0 \Rightarrow t=\mathrm{I},
$$

and

$$
\gamma_{\mathrm{m}}=\left[Q_{0}\left(j \lambda_{0}\right)^{m}\right]^{\mathrm{I} /(m+\mathrm{I})}, \quad \gamma_{\mathrm{I}}=\frac{\mathrm{I}}{\rho_{\mathrm{m}}(2 m+\mathrm{I})}\left[\rho_{\mathrm{m}}\left(q_{\mathrm{I}}+2 m \lambda_{\mathrm{I}}\right)-\frac{\mathrm{I}}{\gamma_{\mathrm{m}}}\right],
$$

where $\rho_{\mathrm{m}}=\rho(\eta=0)$.

The restriction $\beta=0$ is the same as for the plane-flow problem.

The appearance of the $\rho$ term in Equation (55) means that the problem cannot be converted to an initial-value problem as before, but must be solved as a two-point boundary-value problem. Equation (55) can be written as two first-order ordinary differential equations for $\eta, \rho$ with $\gamma_{0}$ as the independent variable:

where

$$
\left.\begin{array}{l}
\frac{\mathrm{d} \eta}{\mathrm{d} \gamma_{0}}=F_{\mathrm{I}}\left(\rho, \eta, \gamma_{0}\right) / F_{2}\left(\rho, \eta, \gamma_{0}\right), \\
\frac{\mathrm{d} \rho}{\mathrm{d} \gamma_{0}}=\frac{\mathrm{I}}{\gamma_{0}} \frac{\mathrm{d} \eta}{\mathrm{d} \gamma_{0}},
\end{array}\right\}
$$

$$
\begin{gathered}
F_{\mathrm{I}}\left(\rho, \eta, \gamma_{0}\right)=m \eta\left(-\frac{\gamma_{0} \eta}{j \bar{\Lambda}}\right)^{m}-\gamma_{0} \eta^{3} \Omega^{\prime}\left(-\gamma_{0} \eta\right), \\
F_{2}\left(\rho, \eta, \gamma_{0}\right)=Q^{\star}-\left(-\frac{\gamma_{0} \eta}{j \bar{\Lambda}}\right)^{m}\left[\frac{\eta}{\rho}+\gamma_{0}\left\{\mathrm{I}+m\left(\mathrm{I}-\frac{\eta \lambda^{\prime}}{\lambda}\right)\right\}\right]- \\
-\left(\frac{\eta^{2}}{\rho}+2 \gamma_{0} \eta\right) \Omega\left(-\gamma_{0} \eta\right)+\gamma_{0}{ }^{2} \eta^{2} \Omega^{\prime}\left(-\gamma_{0} \eta\right),
\end{gathered}
$$

and the prime denotes the derivatives with respect to the argument. In the centre, as $\rho, \gamma_{0} \rightarrow 0$

since $l=\mathrm{I}$. Also

$$
\left.\begin{array}{c}
F_{\mathrm{I}}\left(\rho, \eta, \gamma_{0}\right) \rightarrow 0, \\
F_{\mathrm{I}}\left(\rho, \eta, \gamma_{0}\right) / \gamma_{0} \rightarrow \text { finite, }
\end{array}\right\}
$$

$$
F_{2}\left(\rho, \eta, \gamma_{0}\right) \rightarrow Q_{\mathrm{c}}-\left(-\frac{\gamma_{0} \eta}{j \lambda_{\mathrm{c}}}\right)^{m} \frac{\eta_{\mathrm{c}}}{\rho}-\frac{\eta_{\mathrm{c}}{ }^{2}}{\rho} \Omega\left(-\gamma_{0} \eta_{\mathrm{c}}\right)
$$


where $\lambda_{\mathrm{c}}=\bar{\Lambda}\left(\eta=\eta_{\mathrm{c}}\right), Q_{\mathrm{c}}=Q^{\star}\left(\eta=\eta_{\mathrm{c}}\right)$. The limit of $F_{2}$, Equation (69), is bounded for $l \geqslant \mathrm{I}$ in the light of the second of Equations (55) and Equation (6o). However, since $l=\mathrm{I}$ the limit must be evaluated explicitly in order to determine $\mathrm{d} \rho / \mathrm{d} \gamma_{0}$ at $\eta=\eta_{\mathrm{c}}, \rho=\gamma_{0}=0$. As $\rho, \gamma_{0} \rightarrow 0$ the essential behaviour of Equation (48) is

$$
\frac{\mathrm{d}}{\mathrm{d} \rho}\left[\eta_{\mathrm{c}} \rho\left\{-\frac{\eta_{\mathrm{c}} \gamma_{0}}{j \lambda_{\mathrm{c}}}\right\}^{m}+\eta_{\mathrm{c}}{ }^{2} \rho \Omega\left(-\eta_{\mathrm{c}} \gamma_{0}\right)+O\left(\rho^{3}\right)\right]=\rho Q_{\mathrm{c}}+O\left(\rho^{2}\right),
$$

which integrated gives

and so

$$
\frac{\eta_{\mathrm{c}}}{\rho}\left[-\frac{\eta_{\mathrm{c}} \gamma_{\mathrm{o}}}{j \lambda_{\mathrm{c}}}\right]^{m}+\frac{\eta_{\mathrm{c}}{ }^{2}}{\rho} \Omega\left(-\eta_{\mathrm{c}} \gamma_{\mathrm{o}}\right)=\frac{1}{2} Q_{\mathrm{c}}+O(\rho),
$$

$$
F_{2}\left(\rho, \eta, \gamma_{0}\right) \rightarrow \frac{1}{2} Q_{c}
$$

So finally, Equations (67) are integrated numerically using the explicit end-point values

$$
\begin{gathered}
\rho=\gamma_{0}=0: \quad \frac{\mathrm{d} \eta}{\mathrm{d} \gamma_{\mathrm{o}}}=0, \\
\frac{\mathrm{d} \rho}{\mathrm{d} \gamma_{\mathrm{o}}}=\left\{\begin{array}{ll}
-\frac{2}{Q_{\mathrm{c}}}\left[\frac{\eta_{\mathrm{c}}{ }^{2}}{j \lambda_{\mathrm{c}}}+\eta_{\mathrm{c}} \Omega_{0}\right], & m=n=\mathrm{I}, \\
-\frac{2 \eta_{\mathrm{c}}{ }^{2}}{j \lambda_{\mathrm{c}} Q_{\mathrm{c}}}, & m=\mathrm{I}, \quad n>\mathrm{I}, \\
-\frac{2 \eta_{\mathrm{c}} \Omega_{\mathrm{o}}}{Q_{\mathrm{c}}}, & m>\mathrm{I}, \quad n=\mathrm{I}, \\
\frac{\mathrm{d} \eta}{\mathrm{d} \gamma_{\mathrm{o}}}=\frac{\mathrm{I}}{\gamma_{\mathrm{m}} \gamma_{\mathrm{I}}}, \\
\frac{\mathrm{d} \rho}{\mathrm{d} \gamma_{\mathrm{o}}}=-\frac{\mathrm{I}}{\gamma_{\mathrm{m}} \gamma_{\mathrm{I}}},
\end{array}\right\}
\end{gathered}
$$

using the first of Equations (58) and Equation (65), where $\gamma_{\mathrm{m}}$ and $\gamma_{\mathrm{I}}$ are given by Equation (66), and $\left.\Omega_{0}\right|_{n=1}$ for Glen's law and Colbeck and Evans' law respectively is

$$
\Omega_{0}=k, \quad \Omega_{0}=C_{0} \text {. }
$$

The boundary conditions are:

$$
\left.\begin{array}{lll}
\gamma_{\mathrm{o}}=-\gamma_{\mathrm{m}}: & \eta=0, & \rho=\rho_{\mathrm{m}} \text {-unknown, } \\
\gamma_{\mathrm{o}}=0: & \rho=0, \quad \eta=\eta_{\mathrm{c}} \text {-unknown. }
\end{array}\right\}
$$

Solutions for various values of the physical parameters were found using a shooting technique. It was found that numerical convergence hinged on good initial choices of $\rho_{\mathrm{m}}$ and $\eta_{\mathrm{c}}$.

\section{Illustrations}

For comparison between laws with different exponents $n$, all results are expressed in terms of the same dimensionless horizontal coordinate $\rho^{\star}$, based on the scale factor $\epsilon_{\mathrm{I}}=\epsilon(n=\mathrm{I})$, and so, from Equation (44)

$$
\rho^{\star}=\frac{\epsilon_{\mathrm{I}}}{\epsilon} \rho=\theta^{(n-\mathrm{I}) / 2(n+\mathrm{I})} \rho
$$


All calculations adopt the values in Equations ( 15 ) with $a=0.1, q_{\mathrm{m}}=3 \times 10^{-9} \mathrm{~m} \mathrm{~s}^{-1}$, so that $\theta=0.09$. The real slope magnitude $\epsilon$ for different $n$ and $h_{0}$ is shown in table I, M and J. Illustrations are shown for linear $\bar{\Lambda}$ and $Q^{\star}$, namely

$$
\bar{\Lambda}(\eta)=\lambda_{0} \eta, \quad Q^{\star}(\eta)=-Q_{0}\left(\mathrm{I}+Q_{0}\right) \eta .
$$

For comparison with Nye's (1959) profile, the latter is rewritten in terms of the dimensionless coordinates $(\rho, \eta)$, taking $q=q_{\mathrm{m}}, \lambda=\lambda\left(h_{0}\right)$ giving

$$
\left.\begin{array}{c}
\eta^{2(n+2) /(n+\mathrm{I})}+\rho^{(n+3) /(n+\mathrm{I})} j \bar{\Lambda}(\mathrm{I}) \frac{(n+2)}{(n+3)} 2^{(n-1) /(n+1)}=\eta_{\mathrm{c}^{2(n+2) /(n+1)},} \\
\rho_{\mathrm{m}}=\left[\frac{(n+3) 2^{-(n-\mathrm{I}) /(n+3)}}{\bar{\Lambda}(\mathrm{I})(n+2) j}\right]^{(n+\mathrm{I}) /(n+3)} \eta_{\mathrm{c}^{2(n+2) /(n+3)},} i=\theta^{(n-1)(n+3) / 2(n+1)^{2}},
\end{array}\right\}
$$

where $\eta_{\mathrm{c}}$ or $\rho_{\mathrm{m}}$ has to be prescribed.

Comparisons between Nye's solution as given by Equation (79) and the complete smallslope solution are shown in Figure 2 with $Q_{0}=\lambda_{0}=\mathrm{I}$, where the value of $\eta_{\mathrm{c}}$ in Equation (79) has been set to that obtained by the analysis presented here.

Figure 3 shows the profiles obtained for four sets of $\left(\lambda_{0}, Q_{0}, m\right)$ using the Colbeck and Evans polynomial law. These profiles are analogous to those shown in figure $3, \mathrm{M}$ and $\mathrm{J}$, and it is clear that the effects of changing the parameters $\left(\lambda_{0}, Q_{0}, m\right)$ are similar for the plane flow and axisymmetric cases. Values of $\rho_{\mathrm{m}}{ }^{\star}$ and $\eta_{\mathrm{c}}$ for $\left(\lambda_{0}, Q_{0}\right)=\mathrm{I}, 5$, Io with $m=\mathrm{I}$, are shown in Table I (cf. M and J, table II).

It now remains to compare the plane-flow and axisymmetric solutions. Plane-flow analysis is simpler and other physical solutions, such as a non-symmetric ice sheet, may be considered. However, by looking at the axisymmetric case, some insight into the effect of a third dimension can be obtained. Nye (1959) predicted a decrease in the aspect ratio of

$$
\left(\mathrm{I}-2^{-\mathrm{I} /(2 m+\mathrm{I})}\right) \times 100 \%= \begin{cases}2 \mathrm{I} \%, & m=\mathrm{I}, \\ \mathrm{II} \%, & m=2.5 .\end{cases}
$$

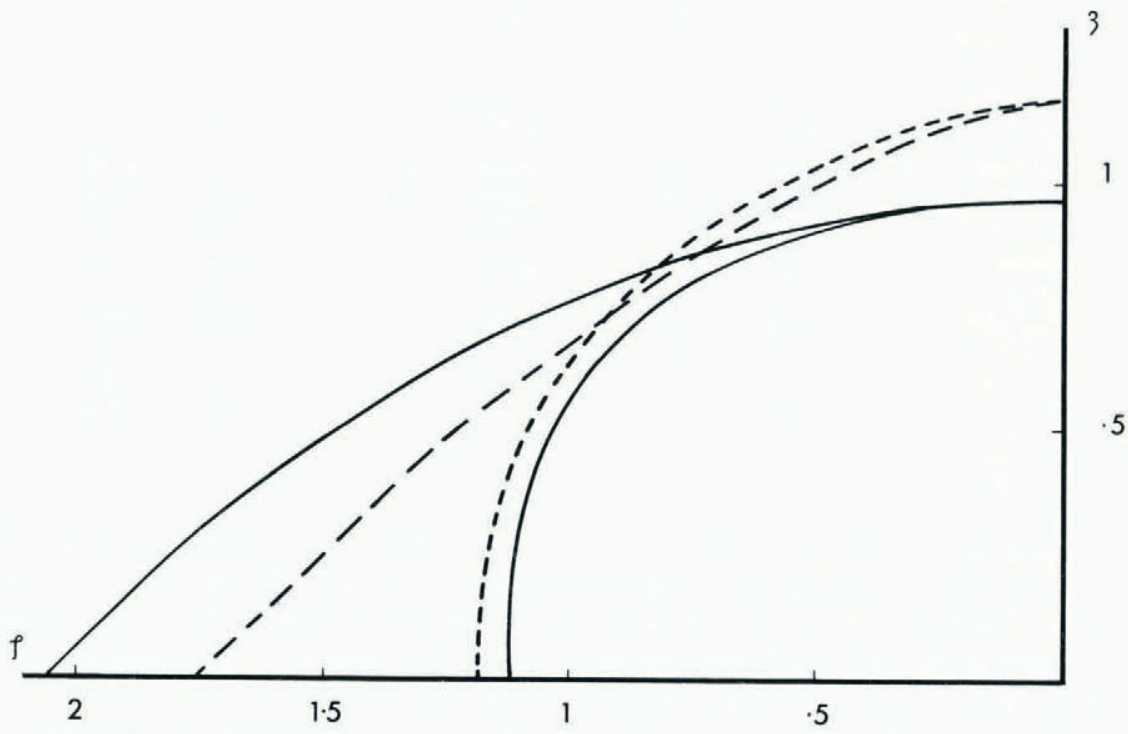

Fig. 2. Comparison of Nye's solution with the complete small-slope profile for Glen's law with linear $\bar{\Lambda}$ and $Q^{\star}$, with $\lambda_{0}=Q_{0}=I ;(i) \stackrel{1}{\longrightarrow} m=I, n=I,(i i)----m=2, n=3$. 


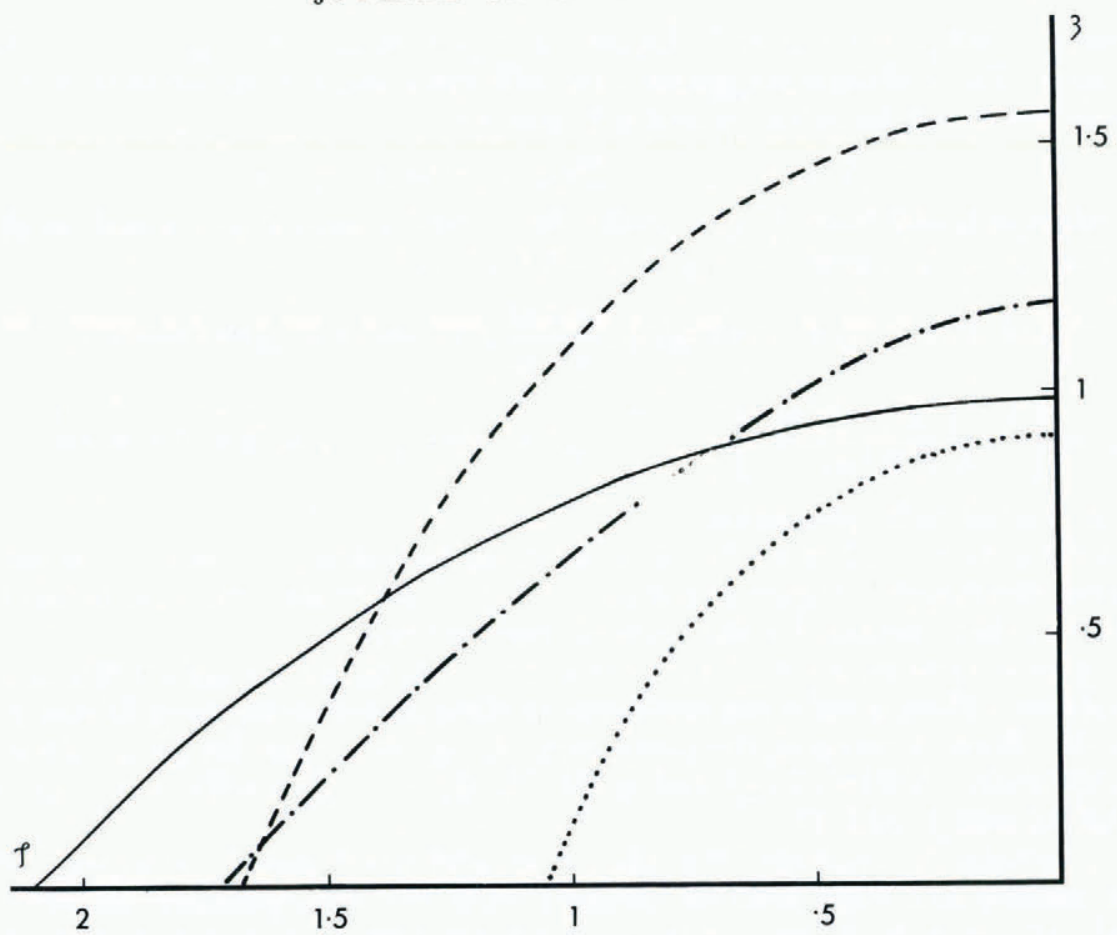

Fig. 3. Comparison of profiles for Colbeck and Evans' law with linear $\bar{\Lambda}$ and $Q^{\star} ;(i)-\lambda_{0}=I, Q_{0}=I, m=I$, (ii) $-\cdot--\lambda_{0}=I, Q_{0}=5, m=I,($ iii $) \cdots \cdots \cdot \lambda_{0}=5, Q_{0}=I, m=I,(i v)-\cdot \cdot \cdot \cdot \lambda_{0}=I, Q_{0}=I, m=3$.

TABle I. Radius $\rho_{\mathrm{m}}{ }^{\star}$ AND HeIGHT $\eta_{\mathrm{c}}$ FOR LiNEAR AGCUMUlation $Q^{\star}(\eta)$ AND LineaR SLIDing LAW $(m=\mathrm{I})$ WITH Linear COEFficient $\bar{\Lambda}(\eta)$. Solutions for Colbeck and Evans' LaW (CE) and Glen's LaW With $n=\mathrm{I}, 3$

\begin{tabular}{|c|c|c|c|c|c|c|}
\hline \multirow{2}{*}{$\begin{array}{r}\lambda_{0} \\
L a w\end{array}$} & \multicolumn{2}{|c|}{ I } & \multicolumn{2}{|c|}{5} & \multicolumn{2}{|c|}{10} \\
\hline & $\rho_{\mathrm{m}}{ }^{\star}$ & $\eta_{\mathrm{c}}$ & $\rho_{\mathrm{m}}{ }^{\star}$ & $\eta_{\mathrm{c}}$ & $\rho_{\mathrm{m}}^{\star}$ & $\eta_{\mathrm{c}}$ \\
\hline $\begin{array}{c}\text { CE } \\
\text { I } \\
3\end{array}$ & $\begin{array}{l}2.071 \\
2.057 \\
1.991\end{array}$ & $\begin{array}{l}0.971 \\
0.976 \\
1.000\end{array}$ & $\begin{array}{l}1.041 \\
1.013 \\
0.905\end{array}$ & $\begin{array}{l}0.905 \\
0.918 \\
0.993\end{array}$ & $\begin{array}{l}0.821 \\
0.785 \\
0.661\end{array}$ & $\begin{array}{l}0.866 \\
0.879 \\
0.978\end{array}$ \\
\hline $\begin{array}{c}\text { CE } \\
\text { I } \\
3\end{array}$ & $\begin{array}{l}\text { I. } 647 \\
\text { г.607 } \\
\text { г.519 }\end{array}$ & $\begin{array}{l}1.555 \\
1.574 \\
\text { I. } 649\end{array}$ & $\begin{array}{l}0.967 \\
0.878 \\
0.804\end{array}$ & $\begin{array}{l}1.413 \\
1.43^{2} \\
1.55^{2}\end{array}$ & $\begin{array}{l}0.840 \\
0.729 \\
0.669\end{array}$ & $\begin{array}{l}\text { I. } 359 \\
\text { I.365 } \\
\text { I. } 501\end{array}$ \\
\hline $\begin{array}{c}\text { CE } \\
\text { I } \\
3\end{array}$ & $\begin{array}{l}\text { I. } 309 \\
\text { I.255 } \\
1.205\end{array}$ & $\begin{array}{l}1.678 \\
1.704 \\
1.775\end{array}$ & $\begin{array}{l}0.821 \\
0.702 \\
0.697\end{array}$ & $\begin{array}{l}1.529 \\
1.543 \\
1.650\end{array}$ & $\begin{array}{l}0.735 \\
0.591 \\
0.605\end{array}$ & $\begin{array}{l}1.4^{83} \\
1.472 \\
1.601\end{array}$ \\
\hline
\end{tabular}

The analysis presented here and in $\mathrm{M}$ and $\mathrm{J}$ does not yield a simple formula for the change in the aspect ratio, but a comparison between table II ( $M$ and $\mathrm{J}$ ) and Table I confirms a decrease (or no change) in all cases considered, as shown by Table II. These values range from $0-17 \%$ compared with Nye's prediction of $21 \%$. It is, however, apparent that changes in the accumulation rate and the sliding law affect this decrease.

Finally, Figure 4 shows both the plane-flow and axisymmetric profiles on the same horizontal axis for the Colbeck and Evans polynomial law, with $Q_{0}=\lambda_{0}=\mathrm{I}$, and $m=\mathrm{I}$. 
Table II. Percentage decrease in the ASPECT RATIO FROM PLANE TO AXISYMMETRIC FLOW

\begin{tabular}{|c|c|c|c|}
\hline$Q_{0}$ & $\begin{array}{r}\lambda_{0} \\
\text { Law }\end{array}$ & I & 5 \\
\hline \multirow{3}{*}{ I } & CE & 3 & 8 \\
\hline & I & 2 & 7 \\
\hline & 3 & 0 & I \\
\hline \multirow{3}{*}{5} & CE & 6 & I4 \\
\hline & I & 5 & 12 \\
\hline & 3 & 2 & I I \\
\hline \multirow{3}{*}{ IO } & CE & 8 & 15 \\
\hline & I & 6 & 12 \\
\hline & 3 & 4 & 14 \\
\hline
\end{tabular}

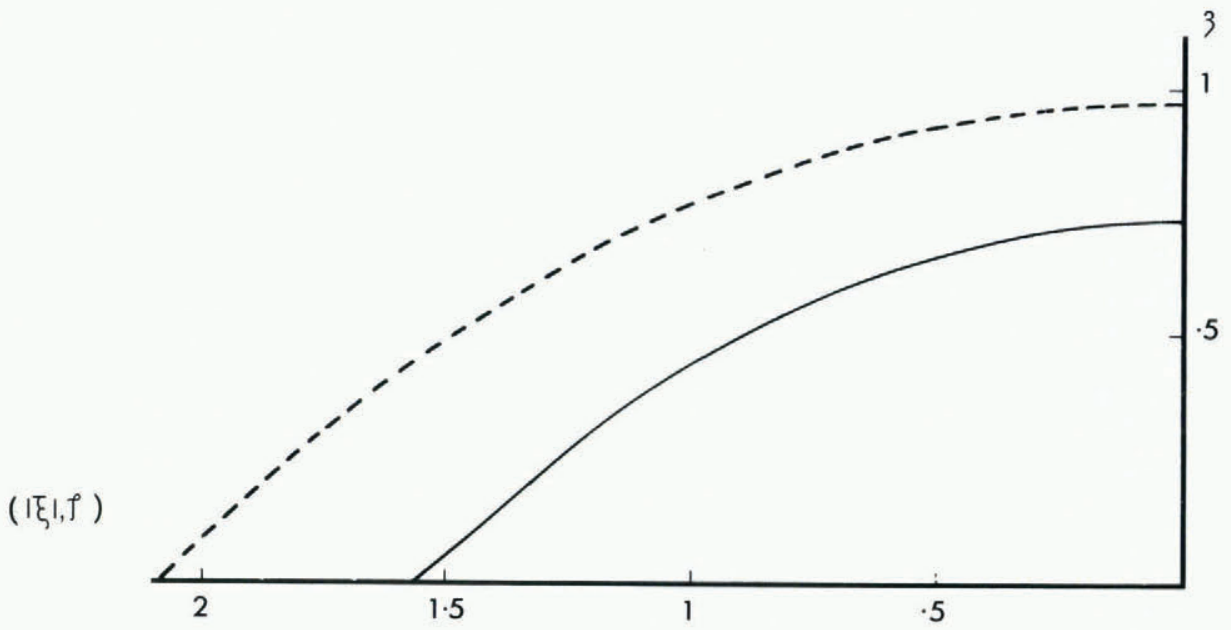

Fig. 4. Comparison between plane and axisymmetric flow for Colbeck and Evans' law with linear $\bar{\Lambda}, Q^{\star}$, where $\lambda_{0}=Q_{0}=I$, and $m=I ;(i) \longrightarrow$ plane flow, (ii) ----- axisymmetric flow.

\section{Acknowledgement}

I am indebted to Dr L. W. Morland for helpful discussions and for reading of the initial draft and to the Science Research Council for the award of a research studentship.

MS. received 13 December 1979

\section{REFERENCES}

Morland, L. W. 1979. Constitutive laws for ice. Cold Regions Science and Technology, Vol. I, No. 2, p. 10 I-o8.

Morland, L. W., and Johnson, I. R. 1980. Steady motion of ice sheets. Journal of Glaciology, Vol. 25, No. 92, p. $229-46$.

Nye, J. F. 1959. The motion of ice sheets and glaciers. Journal of Glaciology, Vol. 3, No. 26, p. $493-507$.

Weertman, J. I96r: Equilibrium profile of ice caps. Journal of Glaciology, Vol. 3, No. 30, p. 953-64. 